\title{
A technique for noise measurement optimization with spectrum analyzers
}

\author{
P. Carniti ${ }^{a, b *}$, L. Cassina ${ }^{a, b}$, C. Gotti ${ }^{a, b}$, M. Maino ${ }^{a, b}$ and G. Pessina ${ }^{a, b}$ \\ ${ }^{a}$ INFN - Istituto Nazionale di Fisica Nucleare, sezione di Milano-Bicocca, \\ Piazza della Scienza 3, Milano, 20126-I, Italy \\ ${ }^{b}$ Università di Milano-Bicocca, Dipartimento di Fisica, \\ Piazza della Scienza 3, Milano, 20126-I, Italy \\ E-mail: paolo.carniti@mib.infn.it
}

\begin{abstract}
Measuring low noise of electronic devices with a spectrum analyzer requires particular care as the instrument could add significant contributions. A Low Noise Amplifier, LNA, is therefore necessary to be connected between the source to be measured and the instrument, to mitigate its effect at the LNA input. In the present work we suggest a technique for the implementation of the LNA that allows to optimize both low frequency noise and white noise, obtaining outstanding performance in a very broad frequency range.
\end{abstract}

KEYWORDS: Instrument optimization; Instrumental noise; Analogue electronic circuits.

${ }^{*}$ Corresponding author. 


\section{Contents}

1. Introduction 11

2. Low noise measurements with spectrum analyzers

3. Low Noise Amplifier optimization for precise noise measurements 5

3.1 Noise measurement configuration 6

3.2 Transfer Function measurement

4. Conclusions

\section{Introduction}

Measuring the noise spectrum of an electronic device is quite a common task for an electronic designer. Spectrum analyzers are used for this purpose but, as it happens very often, the low level of the noise floor from the instrument is not small enough and an unwanted contribution could be added to the measurement result. To mitigate this contribution, a Low Noise Amplifier, LNA, is put between the source to be measured and the instrument. The LNA becomes an important part of the measurement setup [1], 2], as it must show a noise comparable or, better, smaller than the noise of the source to be characterized, and adequate gain and bandwidth. It is very difficult to meet all the specifications at the same time and a compromise is usually considered: a large bandwidth LNA with both good white noise and Low Frequency Noise, LFN, is hard to obtain.

In the following work some suggestions are given trying to meet all the requirements, obtaining a LNA as general purpose as possible.

\section{Low noise measurements with spectrum analyzers}

Noise measurement of an electronic device, DUT or Device Under Test, follows the simplified scheme of Figure 1 with a direct connection to the instrument, the Spectrum Analyzer, SA. In this case the noise from the SA adds (square summed) to the noise of the DUT and it must be at least 5 times smaller for contributing to less than $5 \%$. DUT noise, $n_{D U T}$, is referred to its input or any other node of interest. A test signal that spans the frequency range of interest is connected and the transfer function is measured by the SA. Then the test signal is removed and the noise is measured by the SA. The ratio between the noise and the transfer function gives the input noise:

$$
n_{\text {DUTmeasured }}^{2}=n_{D U T}^{2}+\frac{n_{S A}^{2}}{\left|T_{D U T}(f)\right|^{2}}
$$




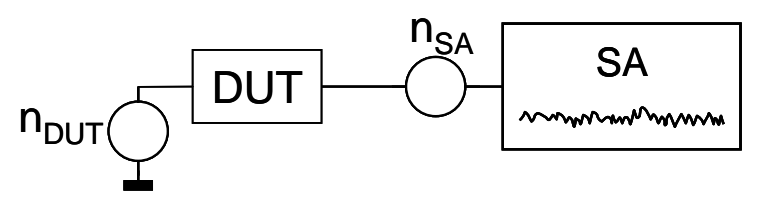

Figure 1. Minimal setup for noise characterization of a DUT. SA is for Spectrum Analyser, $n_{D U T}$ is the DUT noise while $n_{S A}$ is the noise from the SA.

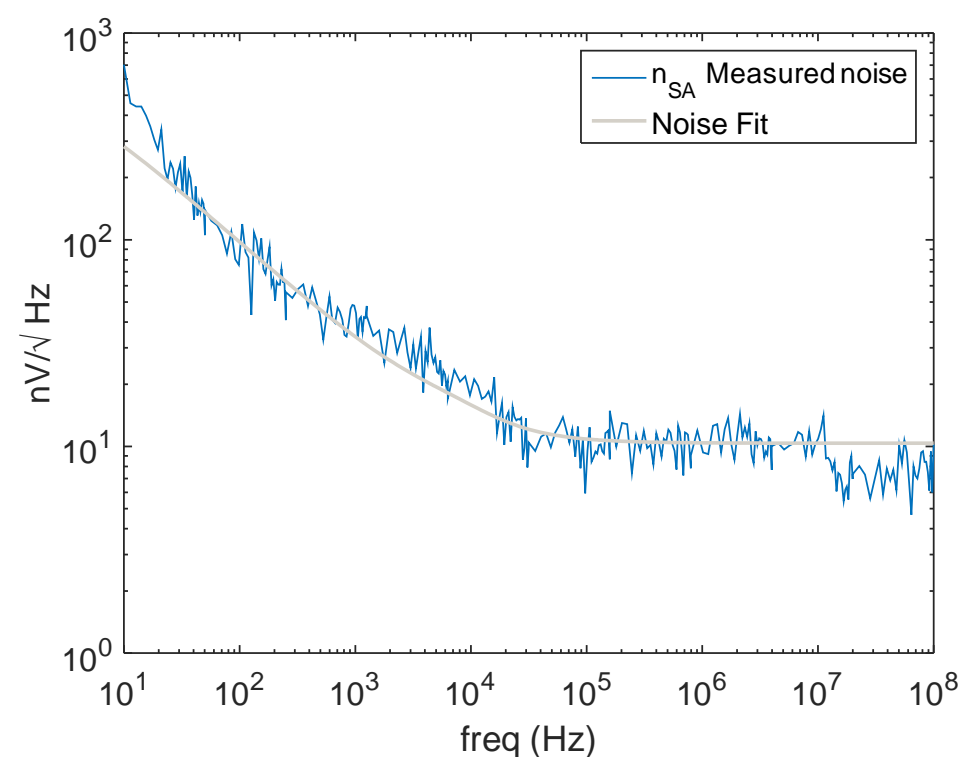

Figure 2. Input noise spectrum of the SA used for this work, Agilent AG4395A.

where $n_{\text {DUT measured }}$ is the actual measured noise at the input, expressed in $n V / \sqrt{H z}$ from now on, which is also contributed by the SA, divided by the DUT transfer function $T_{D U T}(f)$. For simplicity $T_{D U T}(f)$ will be considered equal to one in the following.

Figure 2 shows the noise of the SA used in this work, an Agilent AG4395A in $100 \mathrm{MHz}$ frequency range ( $10 \mathrm{~Hz}$ is the minimum settable frequency). The measurement was taken connecting its input to ground with $50 \Omega$ to match the instrument input impedance. Lines with non-optimal termination degrade the high frequency performance, unless the DUT is connected very close to the input terminal of the instrument. The measurement of Figure 1 shows a noise of a few hundred $n V / \sqrt{H z}$ at $10 \mathrm{~Hz}$ and more than $10 \mathrm{nV} / \sqrt{\mathrm{Hz}}$ white noise. This noise level, very small for such an instrument, is not adequate for characterizing DUTs with a noise floor of a few $n V / \sqrt{\mathrm{Hz}}$. In Figure 2 the noise spectrum is superimposed by its fit, expressed by a function which was found rather general [3, 团]:

$$
n^{2}=\frac{a}{1+\left(\frac{f}{f_{a}}\right)^{e}}+\frac{b}{1+\left(\frac{f}{f_{b}}\right)^{2}}+\frac{c}{f^{\alpha}}+d
$$

where $f$ is the frequency, $a, b, c, d, e, \alpha, f_{a}$ and $f_{b}$ being the fitting parameters. Parameters $e$ and $\alpha$ are introduced in order to take into account non-standard phenomena such as some residual 


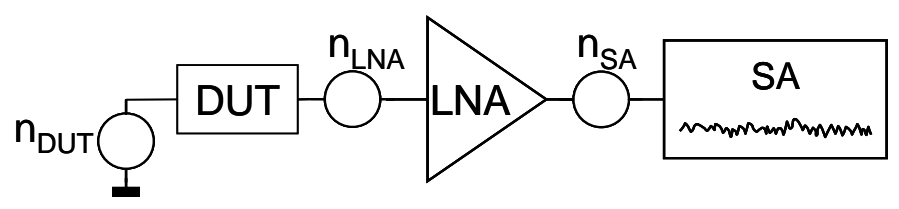

Figure 3. Measurement setup of Figure [1, but with a Low Noise Amplifier, LNA, added between the DUT and the SA to mitigate the noise contribution of the SA.

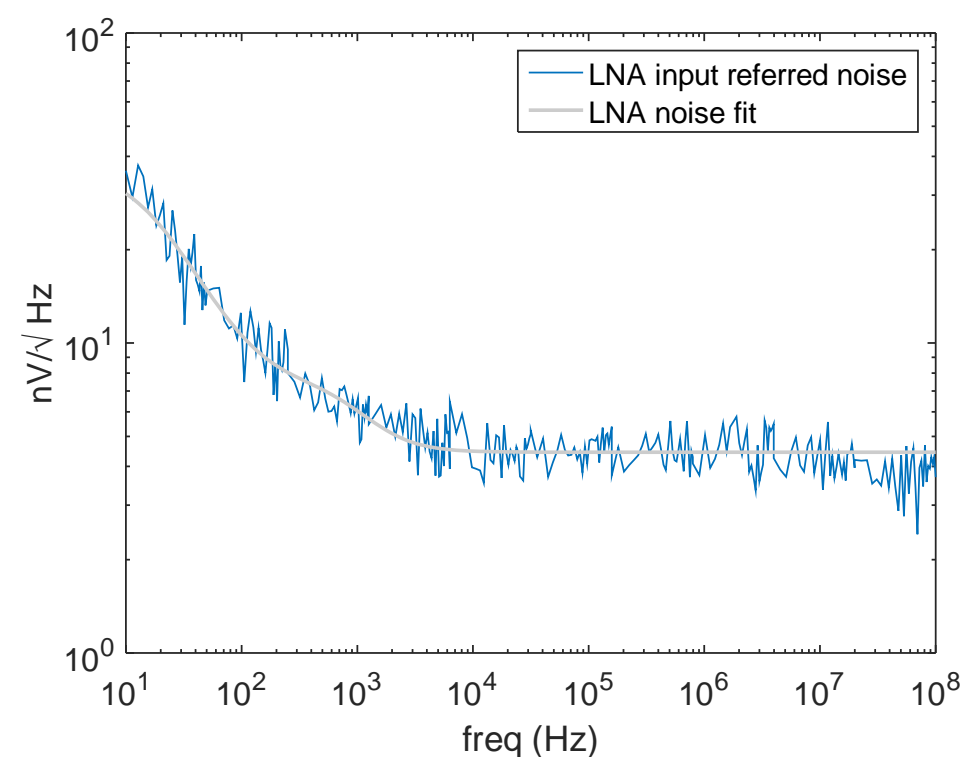

Figure 4. Input referred noise of the OPA657, when employed as the LNA of the setup of Figure B. Noise is now reduced to about $30 \mathrm{nV} / \sqrt{\mathrm{Hz}}$ at $10 \mathrm{~Hz}$ and $5 \mathrm{nV} / \sqrt{\mathrm{Hz}}$ white.

microphonism or low frequency induced disturbances which can cause slight deviations of their values from the usual ones of 2 and 1, respectively.

To mitigate the noise contribution of the SA a Low Noise Amplifier, LNA, is put between the DUT and the SA, Figure 3. Now the DUT noise has to be compared with the LNA input noise $n_{L N A}$, and the SA noise is contributing to $n_{L N A}$ attenuated by the LNA gain. Figure $\theta$ shows the noise spectrum obtained when an OPA657, a large bandwidth Operational Amplifier, OA, is used. LFN is reduced to slightly above $30 \mathrm{nV} / \sqrt{\mathrm{Hz}}$ and white noise is less than $5 \mathrm{nV} / \sqrt{\mathrm{Hz}}$. The OPA657 has a JFET input with a negligible parallel noise, omitted in Figure 3. Noise of Figure 7 is typical of a large bandwidth OA: adequately small white noise floor, but slightly large LFN that, for the case in Figure 4 , is contributed also by SA (larger gains are not recommended for OPA657 as its bandwidth suffers when large value feedback resistors are used).

The noise spectrum in Figure $\$$ was obtained dividing the spectrum, measured with the SA, by the measured and fitted LNA transfer function, TF. The fitting curve is the following:

$$
\mathrm{TF}=B \frac{1}{1+j \frac{f}{f_{2}}} \frac{1}{1+2 j \chi_{3} \frac{f}{f_{3}}-\left(\frac{f}{f_{3}}\right)^{2}} \frac{1}{1+2 j \chi_{4} \frac{f}{f_{4}}-\left(\frac{f}{f_{4}}\right)^{2}}
$$




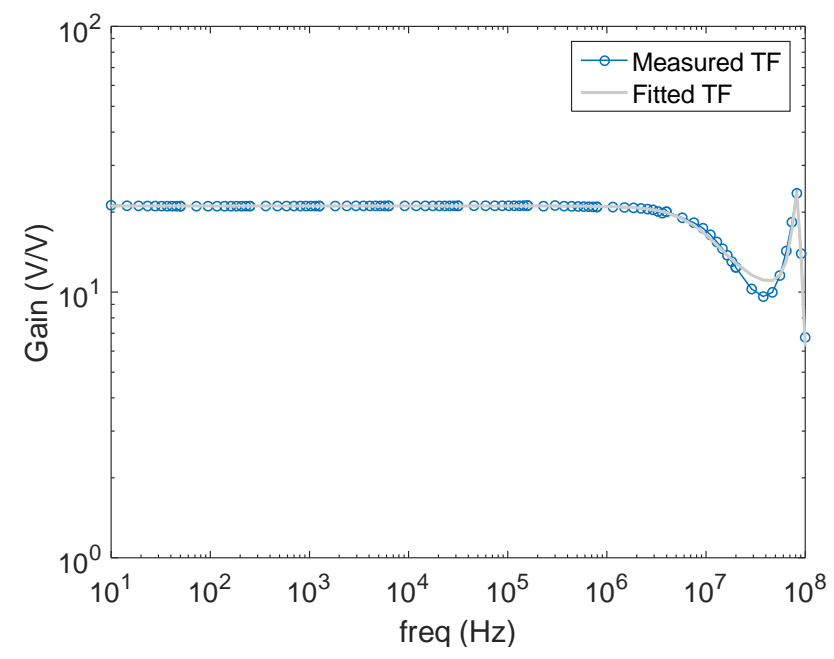

Figure 5. Transfer function, TF, to the LNA input of the circuit of Figure 3.

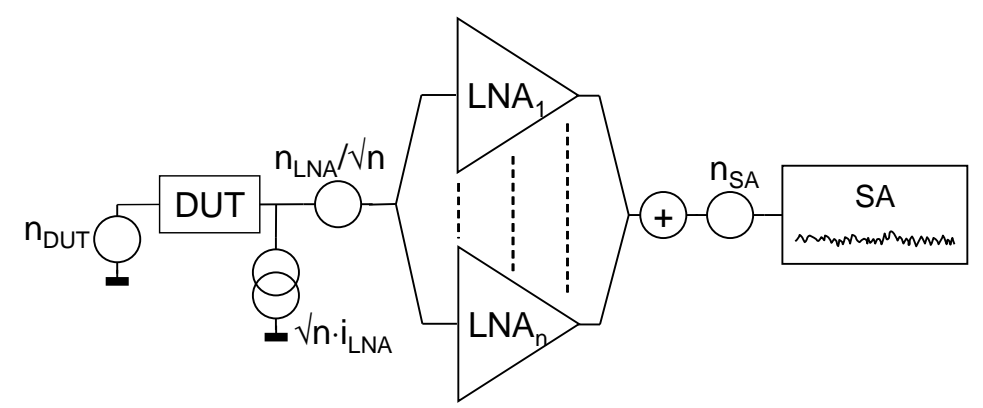

Figure 6. Parallel combination of OAs for obtaining a low LNA series noise. Parallel noise of LNAs is increased and its effect depends on the output impedance of the DUT.

where $B$ is the gain (which includes a factor $\frac{1}{2}$ due to the coaxial line termination at both ends at the SA input), $f_{2}, f_{3}$ and $f_{4}$ are frequencies and $\chi_{3}$ and $\chi_{4}$ consider the complex part of the high frequency poles. We consider 2 pairs of complex poles for redundancy: a good fitting algorithm is able to decide whether it is necessary to mask the contribution of any pole. Figure 5 shows the measured TF and its fit. The result of Figure 4 could be mathematically improved if the SA noise is (square) subtracted from the LNA output before the division by the LNA TF is done. This technique can be applied without adding artifacts only if the SA contribution is adequately small.

In this standard approach, LNAs with bipolar input transistors allow to obtain even smaller white noise, but parallel noise is larger and could contribute if the output impedance from the DUT is not negligible. Current feedback amplifiers, CFAs, have very large bandwidth but cannot be configured with a too large gain because this implies to use large feedback resistors, to which the bandwidth is inversely proportional. Another typical approach is shown in Figure 6 and consists of paralleling several similar LNAs. In this way the resulting series noise is reduced by a factor proportional to the square root of the number of LNAs used. For the circuit having the noise of Figure 4, 9 LNAs are needed to obtain a noise smaller than $10 \mathrm{nV} / \sqrt{\mathrm{Hz}}$ at $10 \mathrm{~Hz}$. Particular care could be needed when using this approach since the parallel noise of the combined LNAs increases 


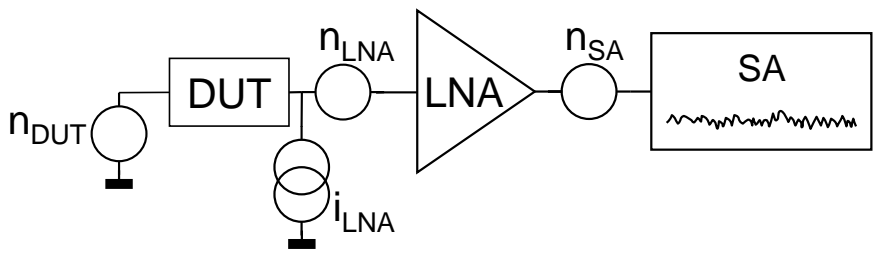

Figure 7. Measurement setup of Figure B without neglecting the parallel noise source.

by the same factor that scales the series noise and this can add noise if the DUT output impedance is not small enough. Further configurations, not based on OAs, exists but they will not be considered here.

The general case which takes also into account high output impedance DUTs can be easily derived from the previous case, by analyzing the circuit shown in Figure 7. The parallel noise $\left(i_{L N A}\right)$ contribution to the input voltage is proportional to the DUT output resistance $R_{O \_} D U T$. The $R_{O_{-} D U T}$ value at which the parallel noise starts to be dominant is $R_{O_{-} D U T}>\frac{n_{L N A}}{i_{L N A}}$. Typical values for series noise of commercial low noise operational amplifiers is of the order of a few $n V / \sqrt{\mathrm{Hz}}$ while parallel noise depends on the type of the input transistors. Low noise FET-input operational amplifiers have an input parallel noise of a few $f A / \sqrt{\mathrm{Hz}}$, while in BJT-input operational amplifiers and CFAs it is a few $p A / \sqrt{H z}$. These numbers roughly give an estimation of which type of operational amplifier could be used with a specific DUT in order to keep the parallel noise contribution negligible: FET-input opamps for output impedance DUTs up to a few hundred $k \Omega$ while CFAs or BJT-input opamps for output impedance DUTs up to a few hundred $\Omega$. It is worth to mention that when noise is characterized on a broad frequency range, the impedances that come into play along the signal path never exceed a few hundred $\Omega$ for not degrading bandwidth due to the presence of parasitic capacitances.

In the following section a very simple approach will be shown, which allows to obtain low noise on the full frequency span, whatever the adopted LNA topology and technology may be.

\section{Low Noise Amplifier optimization for precise noise measurements}

Our suggested approach to the LNA implementation is very simple and based on the following consideration. The noise characterization consists in 2 steps: (a) measurement of the noise at the output of the amplifying chain and (b) measurement of the transfer function of the chain, to scale the noise at the DUT.

The shape of the transfer function is therefore not important as long as it is known (step (b) above). Therefore, differently from the standard use of a flat gain across the whole frequency range, we suggests for the LNA frequency response an optimization with respect to the noise regions, choosing a proper amplifier in each one. LFN can be maintained small with a proper OA choice, which, normally, has a small bandwidth. Combining this OA with a large bandwidth OA, having small white noise, would allow to cover the whole frequency span. The first OA, optimized for LFN, will be given a large gain in order to minimize contributions from both the SA and the second OA in this low frequency region. In the next two subsections details and results are given. 


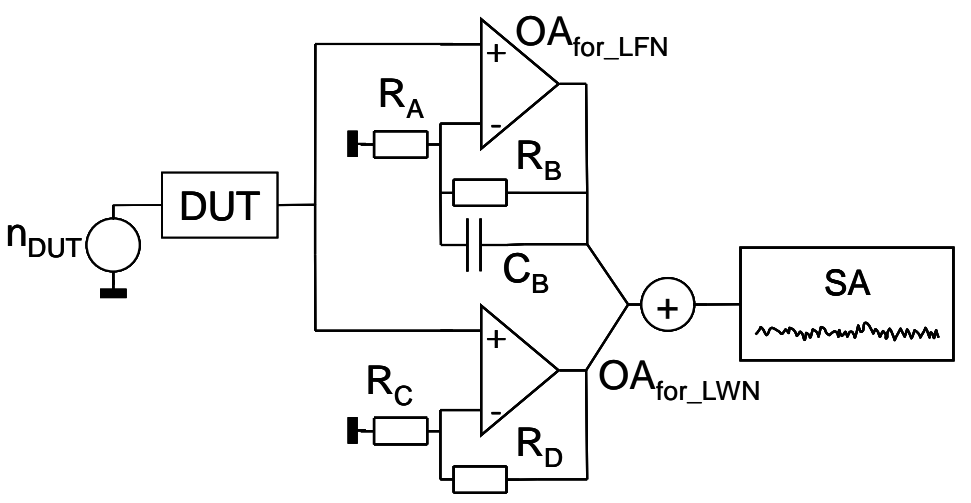

Figure 8. New suggested optimized configuration for noise measurements.

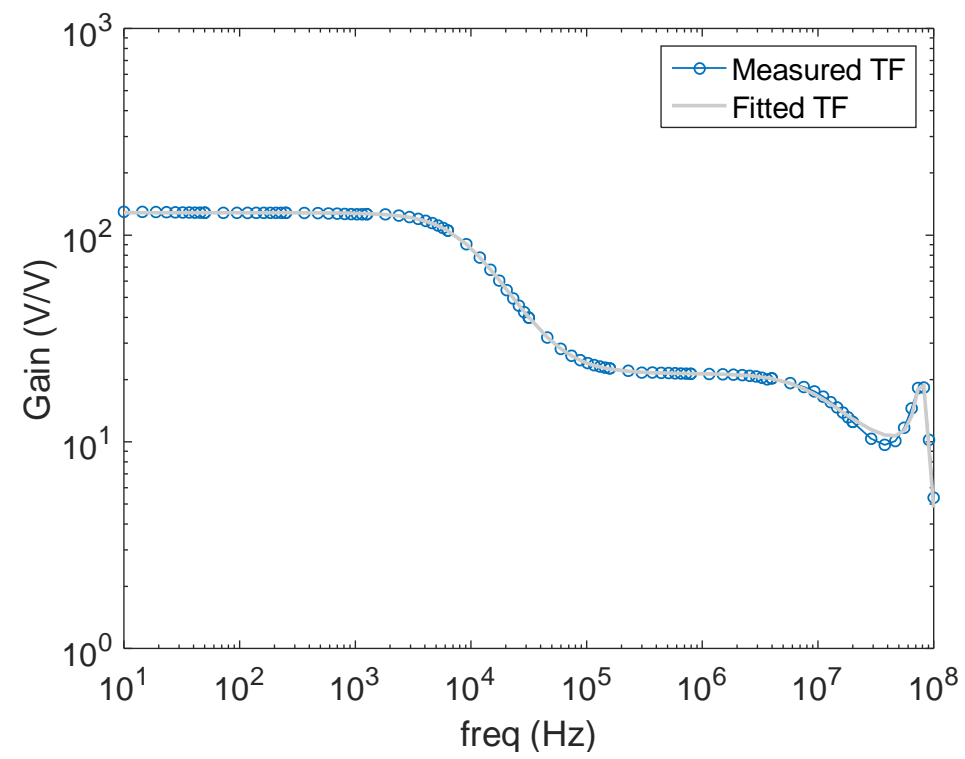

Figure 9. Measured and fitted TF of the circuit of Figure 8.

\subsection{Noise measurement configuration}

The schematic diagram of the suggested optimized configuration is shown in Figure 8. Two OAs, $O A_{\text {for_LFN }}$ and $O A_{\text {for_LWN }}$ (LWN is for Low White Noise), in non-inverting configuration, read the same input node, the DUT, and have the outputs summed at the SA. The summing node is simply an additional OA in inverting configuration. $O A_{\text {for } \_F N}$ is a LFN OA, an OPA140 in this implementation. Its gain is set by $\left(R_{B} / R_{A}+1\right)$ to $201 \mathrm{~V} / \mathrm{V}\left(R_{A}=100 \Omega, R_{B}=20 \mathrm{k} \Omega\right), O A_{\text {for_LWN }}$ is the OPA657 with $\left(R_{D} / R_{C}+1\right)$ set to $40 \mathrm{~V} / \mathrm{V}\left(R_{C}=100 \Omega, R D=3.9 \mathrm{k} \Omega\right)$. OA $A_{\text {for_LFN }}$ has a bandwidth of a few $\mathrm{MHz}$ (with a dominant pole roll-off), while $O A_{f o r} L W N$ has a bandwidth in the $\mathrm{GHz}$ range. Both OAs have JFETs as input transistors. As it can be seen in Figure 9, now the TF is not flat, but shows a different gain dependent on the frequency region and the fitting curve of equation 2.3 becomes: 


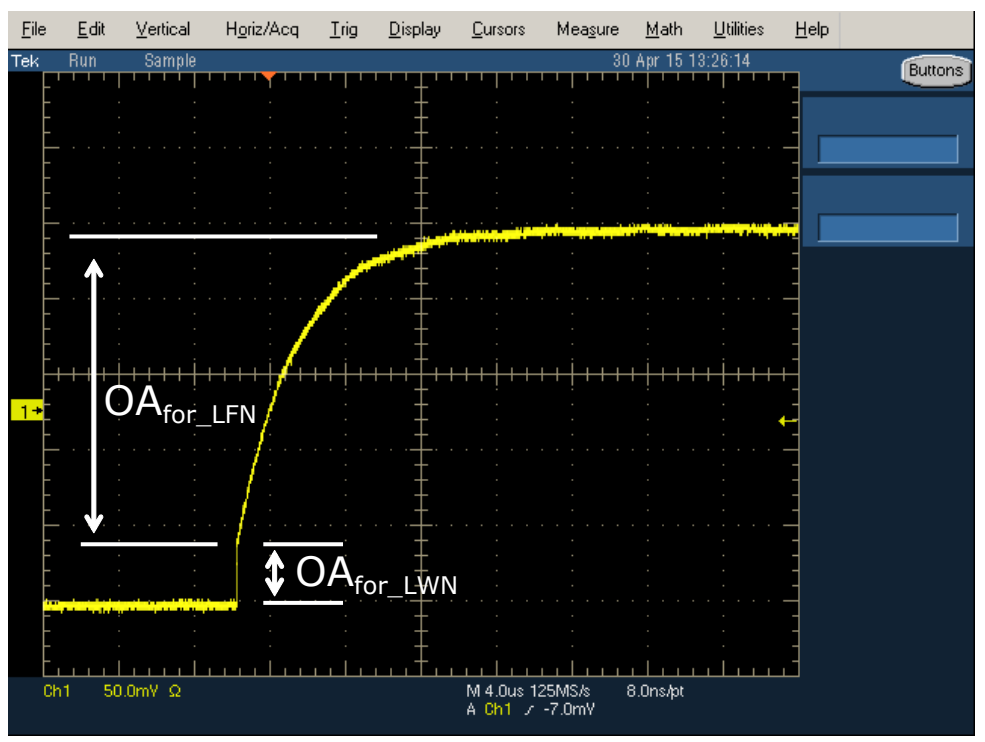

Figure 10. Time domain response of the circuit of Figure 8. The fast transient is due to $O A_{f o r} L W N$, while the slower transient is due to $O A_{f o r} L F N$. The ratio of their amplitudes is related to the chosen gains.

$$
\mathrm{TF}=A \frac{1}{1+j \frac{f}{f_{1}}}+B \frac{1}{1+j \frac{f}{f_{2}}} \frac{1}{1+2 j \chi_{3} \frac{f}{f_{3}}-\left(\frac{f}{f_{3}}\right)^{2}} \frac{1}{1+2 j \chi_{4} \frac{f}{f_{4}}-\left(\frac{f}{f_{4}}\right)^{2}}
$$

Compared to equation 2.3 now there is an additional term that accounts for the added stage. The TF has two frequency regions, one at $f<f_{1}$ where gain is $\left|\mathrm{TF}_{l f}\right|=A+B$ and one at higher frequency where gain is $\left|\mathrm{TF}_{h f}\right|=B$.

At low frequencies the measured DUT noise is:

$$
\left.n_{\text {DUTmeasured }}^{2}\right|_{l f}=n_{D U T}^{2}+\frac{A^{2}}{(A+B)^{2}} n_{O A_{\text {for_LFN }}}^{2}+\frac{B^{2}}{(A+B)^{2}} n_{O A_{\text {for_LWN }}}^{2}+\frac{1}{(A+B)^{2}} n_{S A}^{2}
$$

Since the gain at low frequency is much higher than the one at high frequency $(A+B \gg B)$, the terms depending on $n_{O A_{f o r}{ }_{L W N}}$ and $n_{S A}$ can be neglected and the LFN at the input is dominated only by the $O A_{f o r} L F N$ noise. At high frequency the first term of TF is negligible and $B$ becomes the dominant gain. The measured noise can be expressed with the following equation:

$$
\left.n_{\text {DUTmeasured }}^{2}\right|_{h f}=n_{D U T}^{2}+n_{O A_{\text {for_LWN }}}^{2}+\frac{1}{B^{2}} n_{S A}^{2}
$$

The time response of the circuit to a step is shown in Figure 10. Its noise spectrum is in Figure 11. Now at $10 \mathrm{~Hz}$ the measured noise is close to the white noise, a noticeable improvement with respect to that obtained with the standard approaches (Figures $\mathrm{Z}$ and 4 ).

Going back to the configuration of Figure 8 , it is worth to remark the meaning of capacitor $C_{B}(820 \mathrm{pF})$. This capacitor is added to cut the bandwidth of $O A_{\text {for_LFN }}$ at $10 \mathrm{kHz}$, the frequency region needed for canceling the $\mathrm{LFN}$ contribution from $\mathrm{SA}$ and $O A_{f o r} L W N$. If $C_{B}$ was missing, $O A_{\text {for_LFN }}$ would also contribute with its white noise and the resulting noise spectrum would be the 


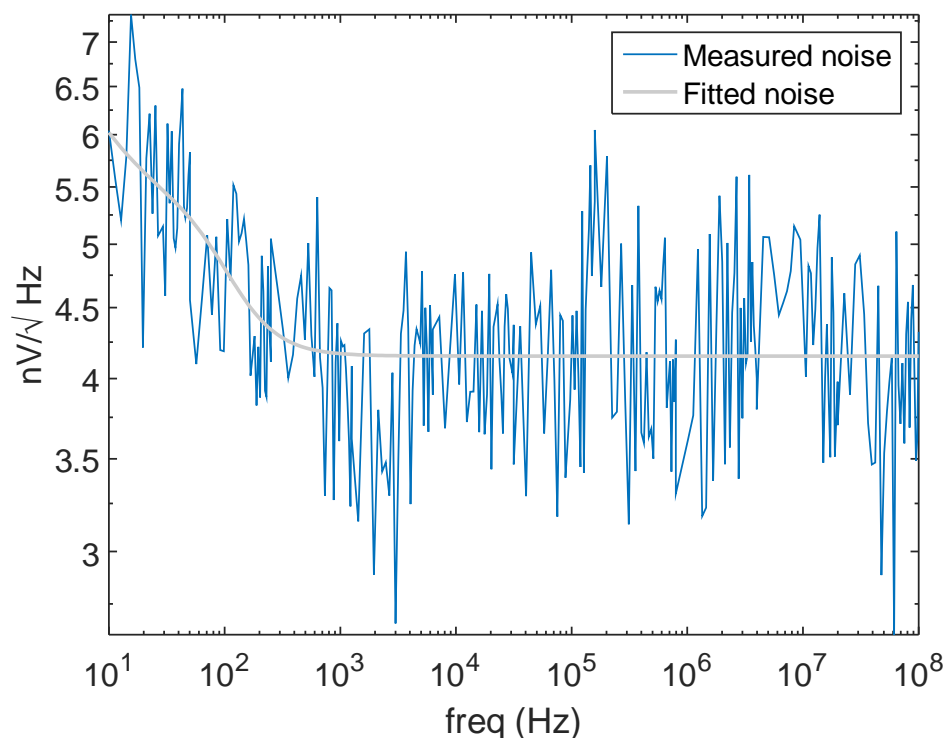

Figure 11. Measured and fitted input noise for the new circuit of Figure 8 .

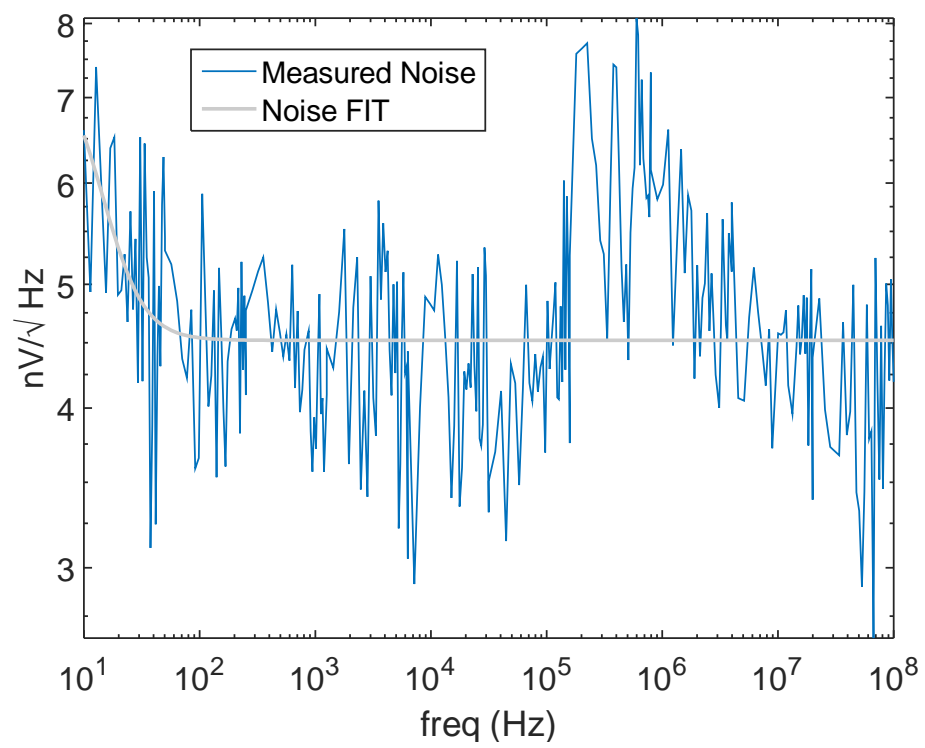

Figure 12. Noise spectrum of the circuit in Figure 8, but without capacitance $C_{B}$. The increase of white noise at about $1 \mathrm{MHz}$ is due to $O A_{\text {for } \angle F N}$, OPA140.

one shown in Figure 12, with an increase in the white noise value around $1 \mathrm{MHz}$. The responsibility for this increase is due to the OPA140 $\left(\mathrm{OA}_{f_{\text {or_ }} L F N}\right)$ a rail-to-rail, r-t-r, output OA. We have noticed that several of this class of OAs suffer from this effect that starts when the closed loop output impedance increases. This is due to the noise of the output buffer, a complex circuit for a r-t-r OA, which increases its noise contribution as soon as the frequency bandwidth of the OA internal gain stage rolls off. This noise behavior is often not addressed in the data-sheets, but there is a 


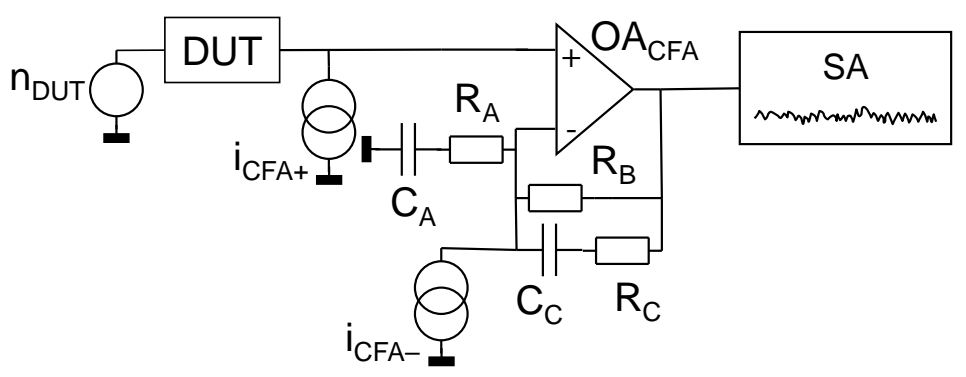

Figure 13. Modified version of the circuit diagram of Figure 8 when a CFA amplifier is used.

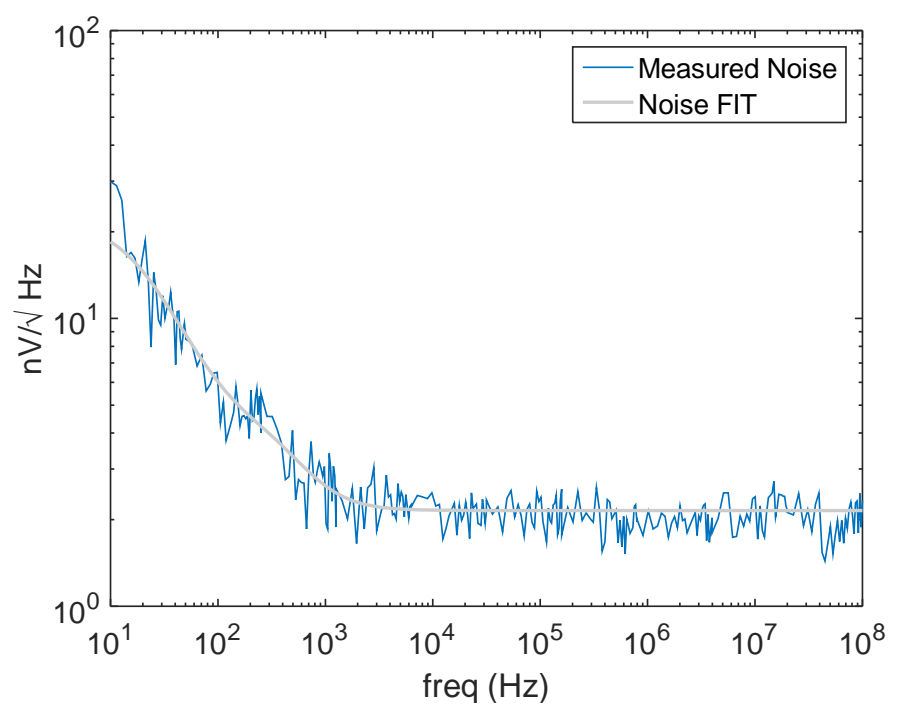

Figure 14. Noise of the circuit of Figure 13 when $C_{C}$ and $R_{C}$ are missing.

way to verify it looking at the two plots, normally included in the data-sheets, that show the series noise and the output impedance versus frequency in closed loop condition: the upper frequency of the span for the former coincides with the frequency at which the output resistance starts to increase. We, therefore, exploited the good LFN characteristic of the OPA140 and canceled its high frequency contribution with $C_{B}$.

A simpler solution for the suggested circuit of Figure 8 can be implemented if the parallel noise has no effect on the output impedance of the DUT. In this case it is possible to take advantage of a particular characteristic of CFAs. This class of devices shows a frequency bandwidth almost independent on the closed loop gain as long as the feedback resistance has a proper value and the resistance connected between the inverting input and ground is large enough. If these constraints are not met, the bandwidth lowers.

Referring to the circuit of Figure [13, the standard configuration is obtained if $C_{C}$ and $R_{C}$ are missing. The settable gain for good performance must be smaller than about $50 \mathrm{~V} / \mathrm{V}$ for the AD8001 adopted in this implementation, a quite general case indeed, and the resulting noise spectrum is shown in Figure 14. As it can be seen the noise at low frequency is also contributed by the one from the SA, resulting in about $20 \mathrm{nV} / \sqrt{\mathrm{Hz}}$ at $10 \mathrm{~Hz}$. 


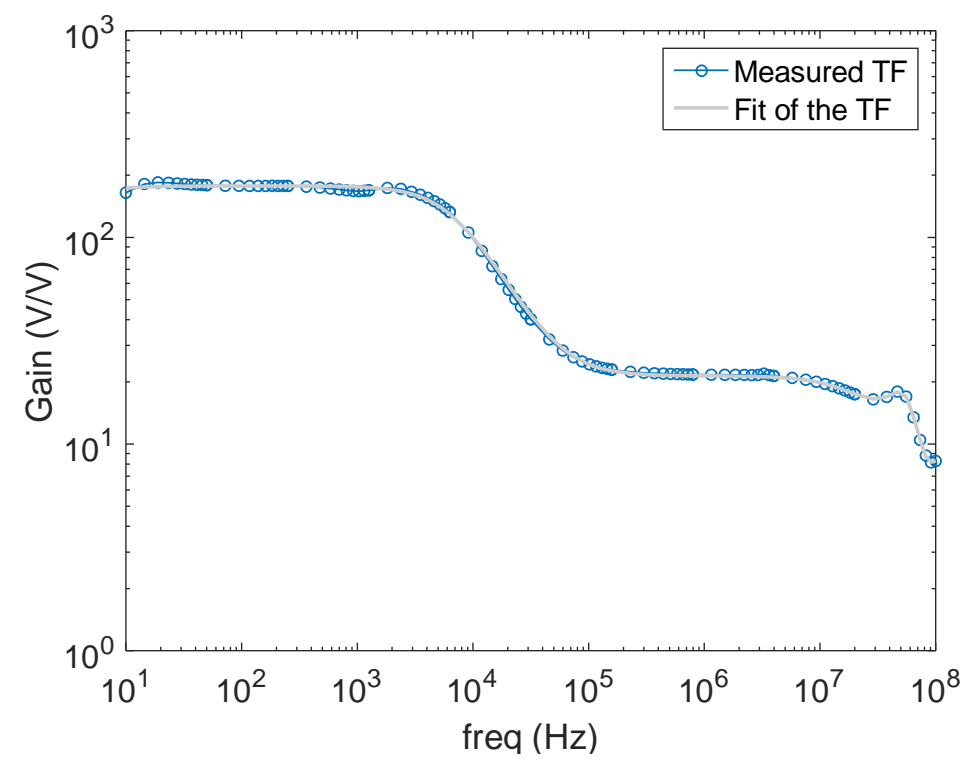

Figure 15. TF of the circuit of Figure 13 for a large value of resistor $R_{B}$ and small high frequency gain.

In this implementation large values for resistors $R_{A}$ and $R_{B}$ are used $\left(R_{A}=10 \Omega, R_{B}=3.6 \mathrm{k} \Omega\right)$ in order to obtain a gain of about $400 \mathrm{~V} / \mathrm{V}$ at low frequencies. Capacitor $C_{C}(3.3 \mathrm{nF})$ and resistor $R_{C}(470 \Omega)$ are set in order to lower the gain at a value of less than $50 \mathrm{~V} / \mathrm{V}$ after a few tens of $\mathrm{kHz}$, using the optimum suggested value for $R_{C}$. The expression of the voltage gain of the circuit of Figure 13 is $(\omega=2 \pi f)$ :

$$
\text { Gain }=1+\frac{R_{B}}{R_{A}} \frac{j \omega C_{A} R_{A}}{1+j \omega C_{A} R_{A}} \frac{1}{1+j \omega C_{C}\left(R_{B}+R_{C}\right)}+\frac{R_{C} \| R_{B}}{R_{A}} \frac{j \omega C_{A} R_{A}}{1+j \omega C_{A} R_{A}} \frac{j \omega C_{C}\left(R_{B}+R_{C}\right)}{1+j \omega C_{C}\left(R_{B}+R_{C}\right)}
$$

Assuming $R_{B}>>R_{A}, R_{C}$, this equation can be separated in two different frequency domains, one is given by the first term which is dominant below the frequency of the pole given by $C_{C}$ and $R_{B}+R_{C}$ while the other one becomes the dominant contribution above the frequency of the same pole. The first term gives a gain of $\frac{R_{B}}{R_{A}}$ while the second one gives a gain of $\frac{R_{C}}{R_{A}}$. This means that this simpler configuration shown in Figure 13 is topologically equivalent to the one in Figure 8 . Equation 3.4, with the addition of the loop gain roll-off at high frequency, can thus be rewritten in a form similar to equation 3.1:

$$
\mathrm{TF}=\frac{A}{2} \frac{j \frac{f}{f_{0}}}{1+j \frac{f}{f_{0}}} \frac{1}{1+j \frac{f}{f_{1}}}+\frac{B}{2} \frac{1}{1+j \frac{f}{f_{2}}} \frac{1}{1+2 j \chi_{3} \frac{f}{f_{3}}-\left(\frac{f}{f_{3}}\right)^{2}} \frac{1}{1+2 j \chi_{4} \frac{f}{f_{4}}-\left(\frac{f}{f_{4}}\right)^{2}}
$$

The above TF differs from the one in equation 3.1 by the presence of the zero and the pole at $f_{0}$ that accounts for the AC coupling which is added with $C_{A}(2000 \mu \mathrm{F}\|100 \mathrm{nF}\| 10 \mathrm{nF})$ to maintain small the output offset due to the $O A_{C F A}$ input current and offset. The resulting fit of the actual TF is shown in Figure 15, while the noise spectrum for this latter configuration is shown in 


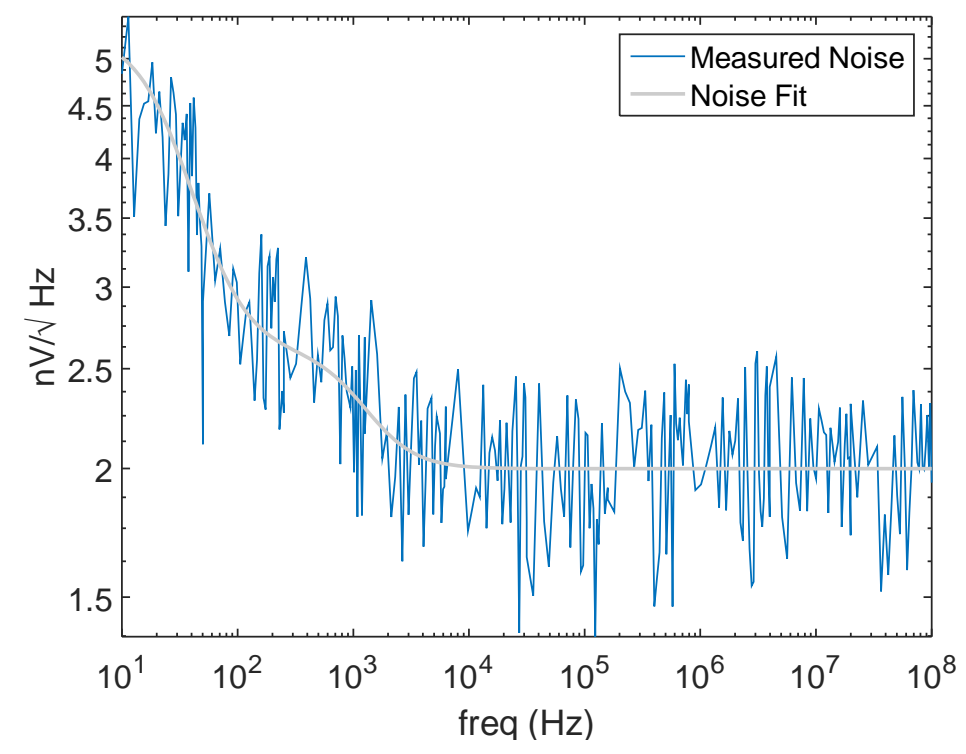

Figure 16. Noise of circuit of Figure 13 with the TF in Figure 15.

Figure 16, again a very remarkable result in the $100 \mathrm{MHz}$ range: noise at $10 \mathrm{~Hz}$ is 5 times lower than in a standard approach.

As previously remarked this simpler solution can be implemented only if the output impedance of the DUT is sufficiently low, so that the higher parallel noise of the CFA can be neglected. This condition is satisfied if the output impedance $R_{O_{-} D U T}$ is lower than the ratio between series noise and parallel noise at the inverting input. Parallel noise at the non-inverting input should not contribute significantly given than $R_{A}$ is chosen low enough (10 $\Omega$ ). In our solution this condition is satisfied if $R_{O_{-} D U T}<\frac{n_{A D 8001}}{i_{A D D 001}}=\frac{2 \mathrm{nV} / \sqrt{\mathrm{Hz}}}{2 \mathrm{pA} / \sqrt{\mathrm{Hz}}}$ (numbers taken from AD8001 datasheet) or $R_{O_{-} D U T}<$ $1000 \Omega$

\subsection{Transfer Function measurement}

The signal to be injected to measure the TF must follow a path as similar as possible to the noise path. In Figure 177 a possible solution to address this is suggested.

The switch $\mathrm{SW}_{1}$ can be implemented with a relay manageable by the instrument itself by means of one of its digital ports (the AG4395A has a few of them and this is a quite common feature for many SAs). If this is the case, it must be guaranteed that disturbances at high frequency are not injected during the noise measurement by the switched-off signal source through the series parasitic capacitance of the switch. In Figure 17 this is avoided thanks to the connection to ground of resistor $R_{S 1}$. A large additional noise suppression factor is given by the attenuation $\frac{R_{S 2}}{R_{S 1}}$, which is set to $\frac{1 \Omega}{51 \Omega}$. Finally, resistor $R_{S 3}$ is needed for increasing the small value of resistor $R_{S 2}$ at the LNA input. This setup does not need the test source to be removed during noise measurement. Alternatively, this signal configuration network can be connected to the other input of the LNA and can be, obviously, used with both the optimized LNAs of Figure 8 and Figure 113. Finally, Figure [17 is configured for an application in which the node for noise characterization is the one at the 


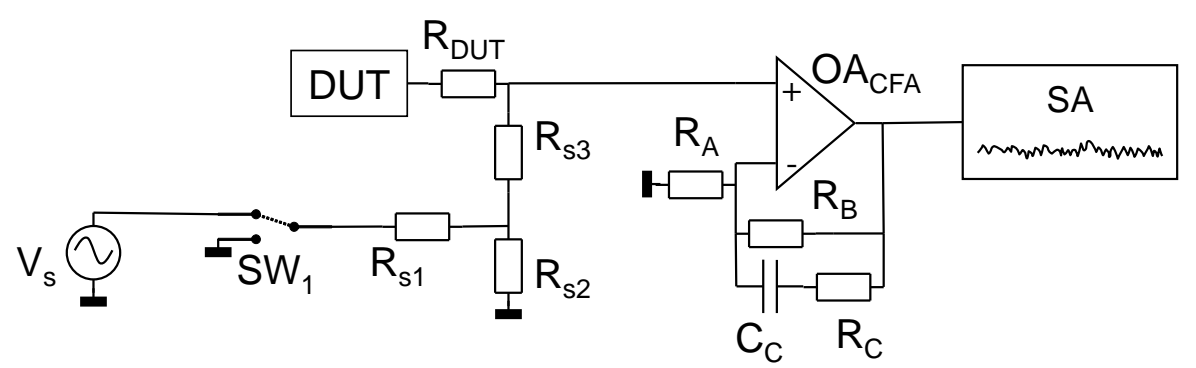

Figure 17. TF measurement configuration.

output of the DUT, like, for instance, when the DUT is a voltage reference or a supply voltage. If the node where the noise is measured is at the DUT input, the configuration is similar and even simpler since resistor $R_{S 3}$ is unnecessary.

\section{Conclusions}

Two circuit configurations for a Low Noise Amplifier implementation in noise measurements with spectrum analyzers are suggested. The very simple yet powerful approach used in these configurations is based on the use of different amplifiers, each one specialized for obtaining its best noise performance in a restricted frequency region. Outputs from these amplifiers are then summed thus obtaining both a wide frequency response and outstanding noise performance. With the method described in this work it was possible to design two Low Noise Amplifiers with an almost flat (white) noise over a frequency span from a few $\mathrm{Hz}$ up to $100 \mathrm{MHz}$.

\section{References}

[1] C.D. Motchenbacher, J.A. Connelly, Low-noise electronic system design, J. Wiley, 1993, chapter 15.

[2] G. Vasilescu, Electronic noise and interfering signals, Springer-Verlag Berlin Heidelberg, 2005, chapter 14.

[3] A. Van Der Ziel, Fluctuation phenomena in semiconductors, London Butterworths Scientific Publications, 1959, chapters 4 and 5.

[4] A. Van Der Ziel, Noise in solid state devices and circuits, John Wiley \& Sons, 1986, chapters 7 and 8. 